\title{
Check on JPL DE405 using modern optical observations
}

\author{
L.V. Morrison and D.W. Evans \\ Royal Greenwich Observatory, Madingley Road, Cambridge CB3 0EZ, UK
}

Received March 9; accepted May 12, 1998

\begin{abstract}
JPL DE405 is compared with optical positions of the outer planets Jupiter to Pluto. Systematic errors in some of the optical observations complicate the interpretation of the residuals between observation and DE405. A common offset in the right ascension of the outer planets suggests a frame-tie problem between DE405 and the ICRF. It is concluded that the overall accuracy of DE405 is better than 50 mas for these planets in the period 19841997.
\end{abstract}

Key words: astrometry — major planets — ephemerides

\section{Introduction}

Ephemerides of the Solar System are generated at JPL by numerical integration of the equations of motion of the planets, the Moon and the more massive minor planets, with initial conditions determined from observations. The initial conditions are refined continuously by the addition of more recent observations, and the resultant ephemerides are issued in the series DExxx, where $\mathrm{xxx}$ is a sequence number. DE405 is the most recent ephemerides to be issued by JPL through their Horizons On-Line Ephemeris System http://ssd.jpl.nasa.gov/cgi-bin/eph interface at their Web site. This is a major departure from the $\mathrm{DE} 2 \mathrm{xx}$ series in the sense that it is referred to the new International Celestial Reference System (ICRS)(Arias et al. 1995). In the ICRS the axes of the international celestial reference frame (ICRF) are fixed by the positions of $\sim 600$ quasars which have absolute positions determined to better than 1 mas by VLBI (Ma et al. 1998) These axes have been aligned to within $\sim 20$ mas (Mignard \& Froeschle 1998) with the previous optical frame, FK5. The major advantage of the ICRS over the previous system is that the axes of the ICRF are fixed (to within $\sim 20 \mu$ as) and there is no rotation at this level.

Send offprint requests to: L.V. Morrison
The orientation of the orbits of the four innermost planets and the Moon have been fixed relative to the ICRF with an accuracy approaching 1 mas using VLBI observations of spacecraft and lunar laser ranging. The relative angles between these objects and their inertial motions have been determined with an accuracy of 1 mas and $20 \mathrm{mas} / \mathrm{cy}$, respectively, using ranging to the Viking Lander spacecraft, radar observations, VLBI and radiometric observations of spacecraft, and lunar laser ranging (Standish 1998). No optical data were used in this linking process because their accuracy is too crude. However, for the outermost planets, Jupiter to Pluto, optical data have been used in generating the numerical integrations because there are either insufficient or no radio data.

The main strength of the optical data is the long timeseries of observations: their main weakness is that the positions are referred to the FK5 frame, or its precursor, the FK4. These frames introduce regional distortions in the sky of 100 mas or more, and the frames have spurious rotations with time, reaching 300 mas/cy. Recently, however, the Hipparcos Catalogue has become available for use in realizing the optical frame. The axes of the Hipparcos Catalogue are aligned with the ICRF to an accuracy of 0.6 mas at the epoch 1991.25 and with a time-dependent part of $25 \mathrm{mas} / \mathrm{cy}$. Modern optical positions of the planets can now be made relative to the ICRF using the Hipparcos and Tycho Catalogues (ESA, 1997).

This paper compares DE405 for the planets Jupiter to Pluto with modern optical positions, either observed with respect to the ICRF via the Hipparcos Catalogue or adjusted retrospectively to the ICRF. This provides a check on the alignment of their orbits relative to the ICRF. In some cases this check is not completely independent because some of the optical positions were used in deriving the starting conditions for the DE405 integration. The degree of circularity in the comparisons will be addressed later in the discussion of the individual planets. 
Table 1. Carlsberg observations of outer Solar System objects analysed in this paper

\begin{tabular}{cccccccc}
\hline Year & Ganymede & Callisto & Titan & Iapetus & Uranus & Neptune & Pluto \\
\hline 1984 & - & - & - & - & 54 & 78 & - \\
1985 & - & - & - & - & 64 & 47 & - \\
1986 & - & 67 & - & - & 101 & 103 & - \\
1987 & - & 26 & 70 & - & 65 & 64 & - \\
$1988-89$ & - & 44 & 89 & - & 105 & 110 & 32 \\
1990 & - & 24 & 40 & 30 & 76 & 105 & 11 \\
$1991-92$ & 24 & 76 & 63 & 59 & 148 & 184 & 107 \\
$1992-93$ & 35 & 28 & 39 & 36 & 57 & 72 & 26 \\
$1994-95$ & 46 & 55 & 26 & 28 & 55 & 66 & 46 \\
$1995-96$ & 69 & 82 & 55 & 55 & 110 & 172 & 100 \\
1997 & 44 & 40 & 21 & 23 & 50 & 101 & 47 \\
\hline
\end{tabular}

\section{Optical observations}

The observations analysed here are taken from four sources: Carlsberg and Bordeaux Automatic Meridian Circles, and the Hipparcos and Tycho Catalogues.

\subsection{Carlsberg Automatic Meridian Circle}

The Carlsberg Automatic Meridian Circle (CAMC) is operated remotely via the Internet on the island of La Palma at the international observatory Roque de los Muchachos of the Instituto Astrofisica de Canarias. It is situated at a latitude of $28.7^{\circ}$ north and an altitude of $2300 \mathrm{~m}$ and is operated jointly by Copenhagen University Observatory, the Royal Greenwich Observatory and the Real Instituto y Observatorio de la Armada, San Fernando. The operating procedure is described in Helmer \& Morrison (1985) and a description of the scanning-slit micrometer and photoelectric detector system can be found in Helmer et al. (1991). The positions of the Solar System objects are measured once nightly as they cross the prime meridian.

The observations discussed in this paper are published in an annual series of catalogues in the period 1985-1997, which have been compiled into one catalogue - Carlsberg Meridian Catalogue Number 10 (1998). The number of observations of Solar System objects in this composite catalogue are listed in Table 1 . The accuracy of the positions is a function of epoch of observation and declination, as listed in Table 2 . The best accuracy of $\pm 0^{\prime \prime} .12$ for a single observation is obtained in the zenith $\left(\right.$ Dec $\left.\sim+30^{\circ}\right)$. The accuracy has improved with time as a consequence of improvements in instrumentation and processing of the raw data.

Before 1995-96 the positions were referred to a frame close to the FK5, as described by Morrison et al. (1990). This is referred to here as the Carlsberg reference frame. The Hipparcos Catalogue has been used to measure the systematic errors of the Carlsberg frame with respect to the ICRF. The observed positions have been corrected retrospectively for these systematic errors. Starting with
Table 2. Accuracy of individual Carlsberg observations

\begin{tabular}{ccccc}
\hline Year & \multicolumn{2}{c}{$\mathrm{RA}$} & \multicolumn{2}{c}{ Dec } \\
& $\delta=+30$ & $\delta=-30$ & $\delta=+30$ & $\delta=-30$ \\
\hline $1984-88$ & $0^{\prime \prime} 19$ & $0^{\prime \prime} 28$ & $0^{\prime \prime} 18$ & $0^{\prime \prime} 134$ \\
$1989-90$ & $0^{\prime \prime} 15$ & $0^{\prime \prime} \cdot 22$ & $0^{\prime \prime} 15$ & $0^{\prime \prime} 27$ \\
$1991-97$ & $0^{\prime \prime} 12$ & $0^{\prime \prime} 19$ & $0^{\prime \prime} 12$ & $0^{\prime \prime} 126$ \\
\hline
\end{tabular}

1995-96 the observations were made with respect to the Hipparcos Catalogue on the ICRF.

In the cases of Jupiter and Saturn, their bright satellites Ganymede, Callisto and Titan and Iapetus were observed, rather than the planets themselves, because of the difficulty of determining the barycentres from scans of their large, non-uniform disks. Lambert scattering was used in reducing the optical positions to the centre of figure (Lindgren 1977). The maximum correction for phase for the planets and satellites discussed in this paper is 50 mas in the case of Ganymede. The choice of scattering law is not critical in this discussion since the consequential variation in position due to different models of the phase correction is always less than $\sim 10$ mas. Corrections for the asymmetry in the albedo of Iapetus are important, however. These have an estimated amplitude in orbital longitude of $\sim 30$ mas with the $\sim 80$-day period of revolution of Iapetus which is tidally locked to Saturn. We return to this point in Sect. 3 .

\subsection{Bordeaux Automatic Meridian Circle}

In 1995 the Bordeaux Automatic Meridian Circle was equipped with a CCD and this made possible the observation of Pluto relative to the Hipparcos/Tycho frame with an accuracy of 70 mas for a single observation (Réquiemè et al. 1997). In the opposition of 1995, 13 observations were made, in 1996 six, and in 1997 13. The mean values and their standard errors were computed for these three oppositions. 


\subsection{Hipparcos/Tycho}

During the Hipparcos mission from 1989 to 1993, 64 and 38 observations of Europa and Titan, respectively, were made by Hipparcos, and 13 and 16 of Callisto and Ganymede, respectively, by Tycho.

Whereas the satellites Callisto and Ganymede were scanned by Tycho using slits inclined at different angles, Europa and Titan were scanned uni-directionally by Hipparcos. The multi-directional scans by Tycho give directly two-dimensional coordinates (right ascension and declination) of Callisto and Ganymede for each observation. The uni-directional observations by Hipparcos, on the other hand, have to be combined in groups having preferably orthogonal directions of scan in order to solve for positions in right ascension and declination. The treatment of the Hipparcos data is given in some detail in Morrison et al. (1997). The three group solutions for Europa and four for Titan given in that paper are used here.

\section{Comparison with DE405}

For Jupiter and Saturn, where two of their satellites were observed, the observations were transferred to the barycentres of the systems using their orbital theories. The ephemeris in the Connaissance des Temps (CdT), which is based on the G-5 theory (Arlot 1982), was used to reduce the observations of the satellites of Jupiter to the barycentre. For Titan and Iapetus, the theories of Taylor \& Shen (1988) and Harper \& Taylor (1993), respectively, were used. The tolerance limit of the ephemeris for Europa is 60 mas, and for Titan it is 50 mas. These should be adequate for the present investigation of possible systematic errors in DE405 because these orbital errors should enter randomly in forming opposition means in right ascension and declination, provided the orbit is well-sampled.

The theory for Iapetus is more complicated because of the importance of solar perturbations, and the accuracy may not be adequate. The asymmetry of the albedo of Iapetus also contributes to the uncertainty of the observed position. For these reasons we investigated the behaviour of the individual Carlsberg observations by plotting the residuals, observation minus theory, as a function of the phase of the orbital period of Iapetus. These are shown in Fig. 1. The amplitude of the fitted sinusoids are $0{ }^{\prime \prime} 10$ and $0^{\prime \prime} 08$ in right ascension and declination, respectively. Whereas the phase of the sinusoids agree with the corrections expected from the asymmetry of the albedo (the dark hemisphere leads), the amplitudes are greater than expected $\left(\sim 0^{\prime \prime} \cdot 03\right)$. The largest contribution is likely to arise from deficiences in the Harper/Taylor theory, and a comparison with the theory of Vienne \& Duriez (1995) could be instructive. From the standpoint of checking the



Fig. 1. Comparison of observations of Iapetus with DE405 and the Harper \& Taylor (1993) theory (upper, right ascension; lower declination). The differences, observation minus DE405+Harper/Taylor, are plotted against the phase of the 79.92-day synodic period of Iapetus, where zero phase is greatest eastern elongation and 0.25 phase is inferior conjunction

DE405 ephemeris of Saturn, we have subtracted these sinusoids from the individual residuals before proceeding. The average offsets in Fig. 1 were retained since these may be interpreted as arising from DE405.

The differences, observation minus DE405, were formed for the four datasets: Carlsberg and Bordeaux opposition means, Hipparcos group solutions and Tycho individual observations. The differences in right ascension and declination and the estimates of the observational errors for Jupiter are plotted in Fig. 2, for Saturn in Fig. 3, for Uranus and Neptune in Fig. 4, and for Pluto in Fig. 5. The estimates of the errors of the Carlsberg opposition means were calculated from the scatter of the individual observations about the means. These do not include possible contributions from systematic errors.

\subsection{Jupiter}

The Hipparcos group solutions in right ascension and declination provide a completely independent check on DE405, since these observations were not used in the integration. The errors of the Hipparcos group solutions lie between 7 and 13 mas, and are thus smaller than the size of the squares used to represent these points in Fig. 2. The individual Tycho observations in declination also provide a good constraint. The greater scatter of the Tycho observations in right ascension is a consequence of the scanning law of the Hipparcos mission.

The optical observations all show a positive bias in right ascension, regardless of the Galilean satellite that was observed. The Hipparcos points for Europa have an average value of +20 mas and the Carlsberg for Callisto and Ganymede an average of +50 mas. In declination the 

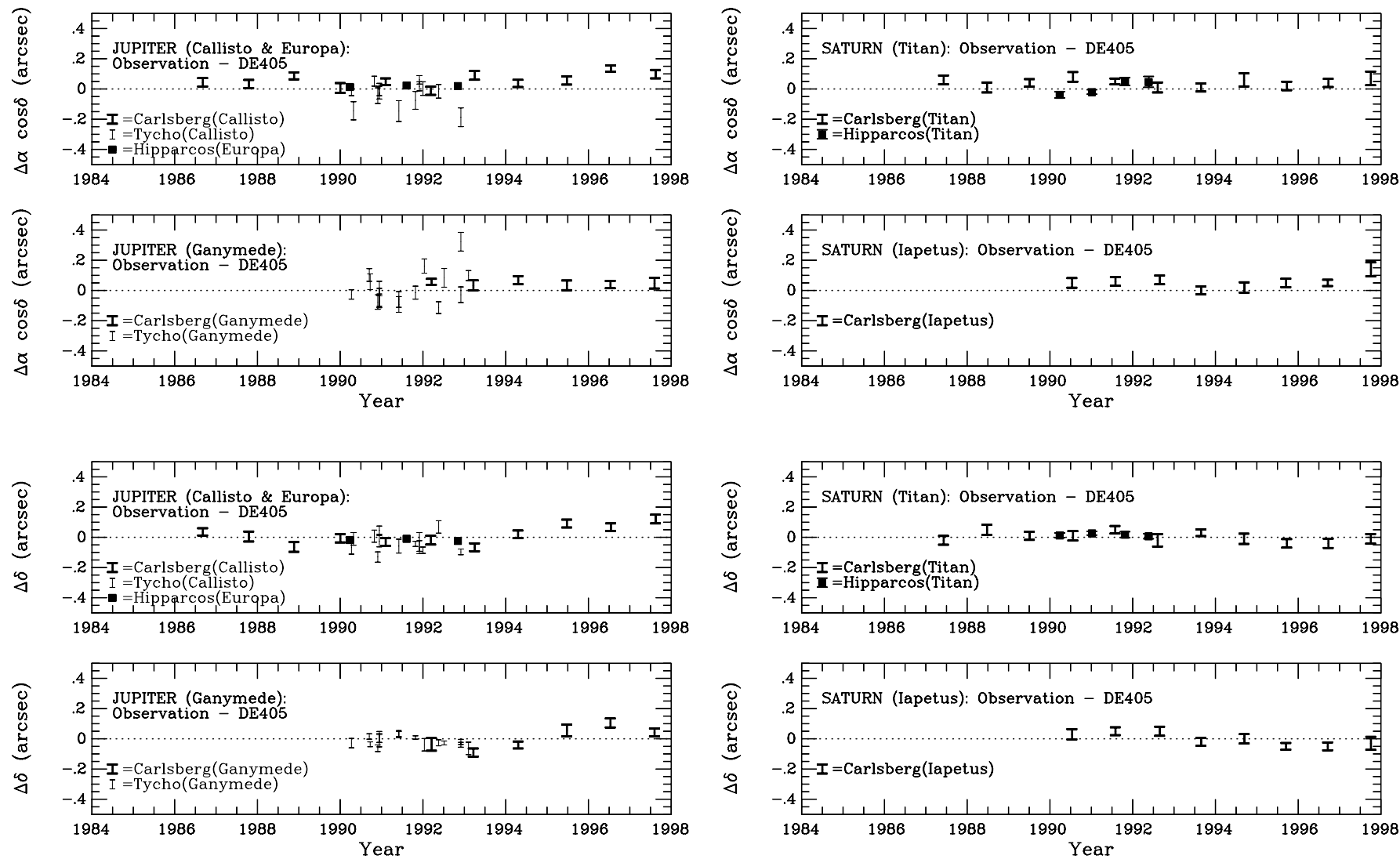

Fig. 2. Comparison of the observation positions of Jupiter with DE405 ephemerides (upper, right ascension; lower, declination). The Hipparcos normal points derived from observations of Europa are shown as filled squares. Individual Tycho positions derived from observations of Callisto are plotted as light error bars. The opposition means derived from Carlsberg observations of Callisto are plotted as heavy error bars

Hipparcos and Tycho observations have an average offset of -20 mas, and the Carlsberg observations show a fluctuation of amplitude $\sim 100$ mas over the 12 -year sidereal period of Jupiter's orbit. The offset in right ascension and fluctuation in declination could result from an offset of the optical reference frame from the radio frame. Only the radio frame was used in fixing the DE405 ephemeris of Jupiter. However, both the optical and radio observations are referred to the ICRF. So the disagreement implies that either the optical or radio observations, or possibly both (for different reasons), are not well linked to the ICRF.

The radio data are not all self-consistent to within their estimated errors. The Voyager 1 data of 1979 and the VLA data of 1983 do not agree with the current ( 1997) VLBI Galileo data (Standish 1998). However, the Galileo data, which are uni-directional, are internally consistent at the level of 10 mas and this engenders a high degree of confidence, especially in the direction of right ascension, to which the data are most sensitive.

The Carlsberg optical data are subject to significant systematic errors, even in the the most recent period



Fig. 3. Comparison of the observation positions of Saturn with DE405 ephemerides (upper, right ascension; lower, declination). The Hipparcos normal points derived from observations of Titan are shown as filled squares with error bars. The opposition means derived from Carlsberg observations of Titan and Iapetus are plotted as heavy error bars

where the positions are measured directly with respect to the Hipparcos frame which is aligned to the ICRF to within 1 mas. This arises because the nightly block adjustment of the instrumental frame is carried out using the observed positions of Hipparcos stars, but this adjustment does not allow for distortions which have a scale length less than about $30^{\circ}$ on the sky. These characteristic distortions can persist for several nights, and thus influence significantly the mean opposition position of a planet which happens to be in that area of the sky. From the discussion of the observations of Uranus and Neptune below, it is estimated that the systematic errors of the opposition means are about \pm 50 mas, and that these systematic errors are not entirely independent from one opposition to the next. This produces spurious fluctuations with a characteristic period of several years which can be seen in all the figures. There is a strong correlation between the fluctuations in the Carlsberg residuals for Callisto and Ganymede, which demonstrates that the fluctuations are independent of any possible shortcomings of the theories of the satellites' orbits. 

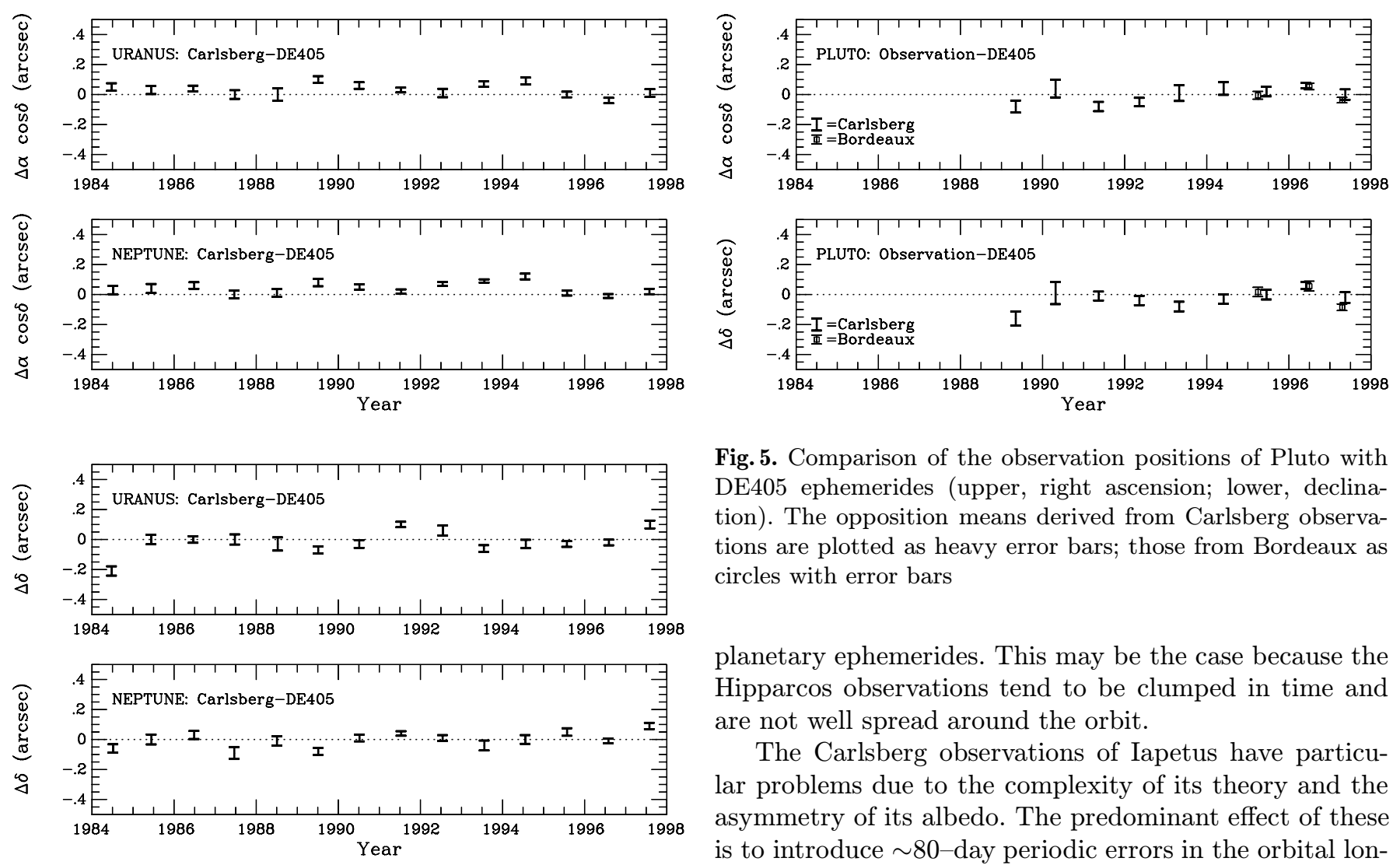

Fig. 5. Comparison of the observation positions of Pluto with DE405 ephemerides (upper, right ascension; lower, declination). The opposition means derived from Carlsberg observations are plotted as heavy error bars; those from Bordeaux as circles with error bars

planetary ephemerides. This may be the case because the Hipparcos observations tend to be clumped in time and are not well spread around the orbit.

The Carlsberg observations of Iapetus have particular problems due to the complexity of its theory and the asymmetry of its albedo. The predominant effect of these is to introduce $\sim 80$-day periodic errors in the orbital longitude which affects the derived position of Saturn. We

Fig. 4. Comparison of the observation positions of Uranus and Neptune with DE405 ephemerides (upper, right ascension; lower, declination). The opposition means derived from Carlsberg observations are plotted as heavy error bars

Notwithstanding, this still does not explain completely the fluctuation in the Carlsberg-DE405 residuals in declination which, suspiciously, has the 12 -year sidereal period of Jupiter's orbit. The most likely explanation for this fluctuation is a misalignment of the radio and optical frames to which the observations are referred.

\subsection{Saturn}

The Hipparcos observations confirm that the accuracy of DE405 for the declination of Saturn is within 20 mas around the epoch 1991. The constraint is not so tight in right ascension, where the residuals range from -40 mas to +50 mas. The apparent internal inconsistency of the Hipparcos points in right ascension could not be removed by changing the starting conditions of the DE405 integration, because any reasonable change would simply move the zero-point vertically in Fig. 3 and would not change the slope around 1991, as required by the observations. This suggests that the errors of the Hipparcos points in right ascension may have been underestimated. This could occur if corrections to the orbital elements of Titan (not considered here) were correlated with corrections to the have corrected empirically for this, but it still may not be completely satisfactory. These observations may be of more use in the improvement in the theory of Iapetus than they are in checking the DE405 ephemeris of Saturn. This said, the Carlsberg observations do show a positive bias in right ascension, similar to that of Jupiter, and, also to those of Uranus and Neptune.

\subsection{Uranus and Neptune}

DE405 is dependent in recent years on the Carlsberg observations; so the overall agreement in Fig. 4 is to be expected. However, the right ascension still shows a positive bias. There is a high correlation between the residuals of Uranus and Neptune, particularly in right ascension. Uranus and Neptune were close together in the sky during the period of the Carlsberg observations. Therefore, the observations of the two planets are subject to the same systematic errors. A possible revision of DE405 could only lead to a smooth linear change in Fig. 4. So, the fluctuations are definitely due to the observations, and their magnitude provides an estimate of the effect of systematic errors on the opposition means which was considered above in the discussion of the Jupiter residuals.

Stone (1998) also reports a positive systematic bias in the right ascension of observations of Uranus and Neptune in 1996 and 1997 taken with the Flagstaff Astrometric Scanning Transit Telescope. The bias is not so pronounced 
for these years in Fig. 4, but the fluctuations due to systematic errors may have reduced the bias fortuitously.

\subsection{Pluto}

DE405 is in good agreement with the Carlsberg and Bordeaux observations, as shown in Fig. 5. Again, this is not surprising because these observations were important in generating DE405. However, they were not the only observations used for this purpose. Another important set was contributed by FASTT at Flagstaff (Stone 1996). The fluctuations in Fig. 5 are due to systematic errors in the Carlsberg observations, as in the case of the other planets.

\section{Conclusions}

The DE405 ephemerides of all the outer planets Jupiter to Pluto are accurate to better than 50 mas in the period 1984-1997.

The Hipparcos and Tycho optical positions show that the DE405 ephemerides for Jupiter (Fig. 2) and Saturn (Fig. 3) are accurate to within $\sim 20$ mas in the period 1991-1993. The Carlsberg observations agree with this conclusion in that period, but outside this period they show a positive bias in right ascension and a fluctuation in the declination of Jupiter with the 12-year orbital period of Jupiter. The correlation between the fluctuations of the residuals for Uranus and Neptune (Fig. 4) leads to the conclusion that the Carlsberg observations of all the planets are subject to systematic errors of a quasi-periodic nature which complicates the detailed interpretation of the residuals. Nevetheless, the common positive bias in the right ascension Jupiter, Saturn, Uranus and Neptune suggests that there is a frame-tie problem in DE405 for the outer planets which requires further investigation.

Using a CCD camera to measure the positions of Solar System objects relative to the ICRF over a field of $\sim 0.3^{\circ}$ is one way of reducing the systematic errors of the optical observations, as demonstrated by Stone (1996) and Réquième et al. (1997). A CCD camera is to be fitted to the Carlsberg telescope in 1998.

Acknowledgements. We thank Daniel Hestroffer for the Tycho observations and Michel Rapaport for supplying the Bordeaux observations.

\section{References}

Arias E.F., Charlot P., Feissel M., Lestrade J.-F., 1995, A\&A 303, 604

Arlot J.-E., 1982, A\&A 107, 305

Carlsberg Meridian Catalogues Number 10, 1998, Copenhagen University Observatory, Royal Greenwich Observatory, Real Instituto y Observatorio de la Armada en San Fernando

ESA 1997, The Hipparcos and Tycho Catalogues, ESA SP-1200

Harper D., Taylor D.B., 1993, A\&A 268, 326

Helmer L., Morrison L.V., 1985, Vista Astr. 28, 505

Helmer L., Fabricius C., Morrison L.V., 1991, Exper. Astr. 2, 85

Lindegren L., 1977, A\&A 57, 55

Ma C., Arias E.F., Eubanks T.M., Fey A.L., Gontier A.-M., Jacobs C.S., Sovers O.J., Archinal B.A., Charlot P., 1998, AJ (in press)

Mignard F., Froeschli M., 1998, Linking the FK5 to the ICRF, in Highlights of Astronomy 11, IAU(23) Joint Discussion 7. Kluwer Acad. Publ., Dordrecht (in press)

Morrison L.V., Argyle R.W., Réquième Y., Helmer L., Fabricius C., Einicke O.H., Buontempo M.E., Muiños J.L., Rapaport M., 1990, A\&A 240, 173

Morrison L.V., Buontempo M.E., 1996, Carlsberg optical astrometry of the outer Solar System, in Dynamics, Ephemerides and Astrometry of the Solar System, FerrazMello S., Morando B., Arlot J.-E. (eds.), IAU Symp. 172, 399. Kluwer Acad. Publ., Dordrecht

Morrison L.V., Buontempo M.E., 1997, Carlsberg positions of planets compared with DE403, in Dynamics and Astrometry of Natural and Artificial Celestial Bodies, Wytrzyszczak I.M., Lieske J.H., Feldman R.A. (eds.), IAU Coll. 165, 541. Kluwer Acad. Publ., Dordrecht

Morrison L.V., Hestroffer D., Taylor D.B., van Leeuwen F., 1997, Check on JPL DExxx using Hipparcos and Tycho observations, in ESA Symposium Hipparcos - Venice 97', 149, ESA SP-402

Réquième Y., Le Campion J.F., Montignac G., Daigne G., Mazurier J.M., Rapaport M., Viateau B., Benevides-Soares P., Teixeira R., Høg E., Makarov V.V., 1997, CCD meridian circle reductions using Tycho positions as reference, in ESA Symposium Hipparcos - Venice 97', 135, ESA SP-402

Standish E.M., 1996, New accuracy levels for Solar System ephemerides, in Dynamics, Ephemerides and Astrometry of the Solar System, Ferraz-Mello S., Morando B., Arlot J.-E. (eds.), IAU Symp. 172, 29. Kluwer Acad. Publ., Dordrecht

Standish E.M., 1998, Linking the dynamical reference frame to the ICRF, in Highlights of Astronomy 11, IAU(23) Joint Discussion 7. Kluwer Acad. Publ., Dordrecht (in press)

Stone R.C., 1996, AJ 112, 781

Stone R.C., 1998, AJ (in press)

Taylor D.B., Shen K.X., 1988, A\&A 200, 269

Vienne A., Duriez L., 1995, A\&A 297, 588 